\title{
Pseudogap, van Hove singularity, maximum in entropy, and specific heat for hole-doped Mott insulators
}

\author{
A. Reymbaut, ${ }^{1,2}$ S. Bergeron, ${ }^{1,2}$ R. Garioud, ${ }^{1,2}$ M. Thénault, ${ }^{2}$ M. Charlebois,,${ }^{1,2}$ P. Sémon, ${ }^{3}$ and A.-M. S. Tremblay $\mathbb{1}^{1,2,4,}{ }^{*}$ \\ ${ }^{1}$ Institut quantique, Université de Sherbrooke, Sherbrooke, Québec, Canada J1K $2 R 1$ \\ ${ }^{2}$ Département de physique and RQMP, Université de Sherbrooke, Sherbrooke, Québec, Canada J1K 2R1 \\ ${ }^{3}$ Computational Science Initiative, Brookhaven National Laboratory, Upton, New York 11973-5000, USA \\ ${ }^{4}$ Canadian Institute for Advanced Research, Toronto, Ontario, Canada M5G $1 Z 8$
}

(Received 6 April 2019; published 13 September 2019)

\begin{abstract}
The first indication of a pseudogap in cuprates came from a sudden decrease of the NMR Knight shift at a doping-dependent temperature $T^{*}(\delta)$. Since then, experiments have found phase transitions at a lower $T_{\text {phase }}^{*}(\delta)$. Using plaquette cellular dynamical mean field for the square-lattice Hubbard model at high temperature, where the results are reliable, we show that $T^{*}(\delta)$ shares many features of $T_{\text {phase }}^{*}(\delta)$. The remarkable agreement with several experiments, including quantum critical behavior of the electronic specific heat, supports the view that the pseudogap is controlled by a finite-doping extension of the Mott transition. We propose further experimental tests.

DOI: 10.1103/PhysRevResearch.1.023015
\end{abstract}

\section{INTRODUCTION}

Below a doping-dependent temperature $T^{*}$, early studies of cuprate high-temperature superconductors found a decrease in the NMR Knight shift [1-5]. This freezing of uniform spin fluctuations, a thermodynamic quantity, became the first signature of what is widely referred to as the pseudogap, one of the remaining challenges for theory. With time, another definition of the pseudogap became more popular. Polarized neutron diffraction [6-8], Nernst effect measurements [9], ultrasound measurements [10], terahertz polarimetry [11], and optical anisotropy measurements [12] report that the prototypical $\mathrm{YBa}_{2} \mathrm{Cu}_{3} \mathrm{O}_{y}$ undergoes a thermodynamic phase transition that breaks time-reversal, spatial inversion, twofold rotational, fourfold rotational, and mirror symmetries below a doping-dependent temperature $T_{\text {phase }}^{*}$ that is distinctly lower than $T^{*}$ at low doping. This suggests that phase transitions are a consequence of the pseudogap first observed in NMR, not the cause $[13,14]$.

In this paper, we address the nature of the pseudogap that was first found in NMR. We focus mostly on thermodynamic signatures at high temperature, where cluster generalizations of dynamical mean-field theory provide a reliable theoretical tool [15]. The remarkable agreement that we find with several experiments supports the view that the high-temperature physics of the pseudogap is controlled by a finite-doping extension of the Mott transition that includes superexchange effects [16]. We propose further experimental tests to investigate that hypothesis. The connection between the Mott

\footnotetext{
*Corresponding author: andre-marie.tremblay@usherbrooke.ca
}

Published by the American Physical Society under the terms of the Creative Commons Attribution 4.0 International license. Further distribution of this work must maintain attribution to the author(s) and the published article's title, journal citation, and DOI. transition and pseudogap was also suggested by calculations in smaller clusters [17].

Although recent experimental results usually relate to the lower-temperature $T_{\text {phase }}^{*}(\delta)$ line, where $\delta$ denotes hole doping, the $T_{\text {phase }}^{*}(\delta)$ and $T^{*}(\delta)$ lines are almost parallel, which suggests that they are related. Experimental results for $T_{\text {phase }}^{*}(\delta)$ can be classified into two families.

The first family of results identifies the main features of the $T_{\text {phase }}^{*}$ line. Recent Hall measurements on $\mathrm{YBa}_{2} \mathrm{Cu}_{3} \mathrm{O}_{y}$ (YBCO), $\mathrm{La}_{2-x} \mathrm{Sr}_{x} \mathrm{CuO}_{4}$ (LSCO), and $\mathrm{La}_{1.6-x} \mathrm{Nd}_{0.4} \mathrm{Sr}_{x} \mathrm{CuO}_{4}$ (Nd-LSCO) highlighted a sharp jump in carrier density with increasing hole doping $\delta$ across a material-dependent critical doping $\delta_{\text {phase }}^{*}$ at which the $T_{\text {phase }}^{*}$ line suddenly drops [18-20]. This drop, also observed in Raman scattering experiments on $\mathrm{Bi}_{2} \mathrm{Sr}_{2} \mathrm{CaCu}_{2} \mathrm{O}_{8+\delta}$ (Bi2212) [21], occurs between a lowdoping antiferromagnetic Mott insulating regime [22] and a high-doping metallic regime [23]. Moreover, the specific heat divided by temperature $C / T$ scales logarithmically as a function of temperature around this sharp transition in LSCO, Nd-LSCO, and $\mathrm{La}_{1.8-x} \mathrm{Eu}_{0.2} \mathrm{Sr}_{x} \mathrm{CuO}_{4}$ (Eu-LSCO) [24], which strengthens its interpretation as a finite-temperature extension of an underlying quantum critical point.

The second family of results regarding the $T_{\text {phase }}^{*}$ line identifies how its main features vary across different materials under external parameters, and how they relate to the rest of the phase diagram. The Nernst effect measurements of Ref. [25] establish that while both the $T_{\text {phase }}^{*}$ line's slope and the position of $\delta_{\text {phase }}^{*}$ differ between different parent compounds (YBCO and LSCO), only the position of $\delta_{\text {phase }}^{*}$ changes with chemical pressure within the same family of compounds (LSCO, Nd-LSCO, and Eu-LSCO). Furthermore, it has been shown in Ref. [26] that $\delta_{\text {phase }}^{*}$ shifts towards lower doping in Nd-LSCO under applied pressure. This unexpected shift seems to be driven by a corresponding shift in $\delta_{\mathrm{VH}}$, the doping at which a van Hove singularity appears in the local density of states. Since this Lifshitz transition in cuprates 
translates into a change of the Fermi surface from holelike to electronlike with increasing doping, as determined by angleresolved photoemission spectroscopy (ARPES) [27-30], this suggests that the pseudogap cannot open on an electronlike Fermi surface, so that the condition

$$
\delta_{\text {phase }}^{*} \leqslant \delta_{\mathrm{VH}}
$$

should always be satisfied. For instance, Ref. [21] reports $\delta_{\text {phase }}^{*} \simeq \delta_{\mathrm{VH}}$ in Bi2212. Apart from the van Hove singularity, experiments performed on calcium-doped YBCO and Bi2212 report a vertical regime of maximum electronic entropy in the phase diagram close to $\delta_{\text {phase }}^{*}$ [31-34]. This observation is consistent with the behavior of thermopower when interpreted in terms of entropy $[35,36]$. Finally, the critical regime around $\delta_{\text {phase }}^{*}$ also corresponds to the zone in the phase diagram where superconductivity is most resilient to strong magnetic fields [37], hinting that it may actually nurture superconductivity. This link between both phases of matter has been the subject of many past studies [25,38-40].

While much is already known about the $T_{\text {phase }}^{*}$ line, much less is known regarding the fate of the NMR $T^{*}$ line with increasing doping, except for the fact that raw data for LSCO are consistent with a sudden drop of $T^{*}$ at a doping $\delta^{*}$ close to $\delta_{\text {phase }}^{*}$ where $T_{\text {phase }}^{*}$ also drops [41].

However, most theoretical works study the $T^{*}$ line, focusing on the two-dimensional single-band Hubbard model on a square lattice [42-44]. In addition, cluster extensions of dynamical mean-field theory (DMFT), such as cellular dynamical mean-field theory (CDMFT) and the dynamical cluster approximation (DCA) [45-47] using various quantum impurity solvers [48-55], have shown that the Hubbard model captures many features of the superconductivity and pseudogap of cuprate compounds [40,47,56-67]. The same applies to its strong-interaction limit when correlated hopping is neglected: the $t-J$ model [68-70]. DCA studies [71,72] found a maximum in entropy and a critical logarithmic scaling for the specific heat $C(T)$ at a critical doping. Moreover, Refs. [67,73,74] established that pseudogap formation and superconducting pairing are both linked through short-ranged spin physics [75-82]. Closer to the aforementioned experiments, Refs. [83,84] found an unambiguous link between pseudogap formation and Fermi-surface topology through the polelike feature of the electronic self-energy, conjecturing that Eq. (1) for $\delta_{\text {phase }}^{*}$ should also hold for $\delta^{*}$.

Finally, a set of CDMFT studies spanning almost a decade has highlighted the existence of a finite-doping phase transition, here dubbed the "Sordi transition" $[16,85]$, that acts as an organizing principle for the $T-\delta$ phase diagram of cuprates $[14,86]$. This transition is a finite-doping extension of the first-order Mott transition found at half filling, separating the pseudogap and correlated metallic phases in the normalstate phase diagram (where superconductivity is not allowed) $[16,85]$. In this normal-state phase diagram, the Sordi transition ends at finite $T$ at a second-order critical point above which it extends as a Widom line [87] in the high-temperature crossover regime. Even if the low-temperature normal-state phase diagram is usually metastable, hidden by more ordered states, the associated high-temperature crossover regime can be observed in a doped organic compound owing to magnetic frustration [88]. More specifically, in both doped Mott insulators $[14,86,89]$ and doped charge-transfer insulators [90], the Knight shift pseudogap $T^{*}$ line is parallel to the Widom line and appears as a high-temperature precursor of this crossover line. $T^{*}(\delta)$ drops to zero precipitously at $\delta^{*}$ in the vicinity of the Sordi transition. In addition, these CDMFT studies retrieve the aforementioned maximum in entropy close to $\delta^{*}[85]$ and a van Hove-like singularity at higher doping [14].

\section{MODEL AND METHODS}

Here, we study the two-dimensional Hubbard model on a square lattice with $t$ the nearest-neighbor hopping, $t^{\prime}$ the next-nearest-neighbor hopping, and $U$ the on-site Coulomb repulsion. The hopping $t=1$ serves as the energy unit, $k_{B} \equiv 1$ and $\hbar \equiv 1$. This model is implemented with CDMFT, where a $2 \times 2$ cluster of sites [61,91] is dynamically coupled to a bath of noninteracting electrons by a frequency-dependent hybridization function. The quantum impurity problem is solved with continuous-time quantum Monte Carlo in the hybridization expansion [51-54]. Note that close to half filling, where finite-size effects are expected to be largest because of antiferromagnetism, systematic studies up to $5 \%$ doping at $T=0.06 t$ show that $2 \times 2$ clusters give accurate results [92] All details regarding our various physical criteria can be found in the Supplemental Material [93].

\section{RESULTS}

\section{A. NMR $T^{*}$, van Hove singularity and maximum of the entropy}

Figure 1 (left) shows the normal-state phase diagram at $t^{\prime}=0$ for $U=9,18$, and 36 . Note that our values of $T^{*}$ for a $2 \times 2$ cluster match the ones found in larger cluster studies with comparable $U$ [94]. The case $U=36$ suffered from severe sign problems but is still useful to capture the evolution of $T^{*}(\delta \rightarrow 0)$ as a function of $U$. Within the Hubbard model, a pseudogap formation arises from the singlet formation due to superexchange, which we retrieve in this figure [14,61]. Indeed, these correlations are well described by the $t-J$ limit of the Hubbard model at strong interaction and low doping, with superexchange $J=4 t^{2} / U$. Going from $U=9$ to $U=$ $18, T^{*}(\delta \rightarrow 0)$ does not actually decrease by a factor of 2 , because the interaction strength is not large enough for the $t-J$ limit to be valid. However, $T^{*}(\delta \rightarrow 0)$ does decrease by a factor of 2 between $U=18$ and $U=36$. In other words, $T^{*}(\delta \rightarrow 0)$ scales with $J[17,68,69]$ for large values of $U$, confirming short-range correlations due to superexchange as the origin of the pseudogap. Furthermore, the order of magnitude of $T^{*}(\delta \rightarrow 0)$ at $U=9$ agrees with the experimental values reported in Refs. $[3,41,95]$ for YBCO and LSCO. If we assume that the value of the Néel temperature is a measure of $J$, this is consistent with the experimental slope of the $T_{\text {phase }}^{*}$ lines of YBCO and LSCO cited in Ref. [25]. Putting $U=36$ aside in Fig. 1 (left), since sign problems appear at low temperature, we find at intermediate doping that the $T^{*}$ line drops suddenly at a critical doping $\delta^{*}$ : The smaller the value of $U$, the larger is the drop of $T^{*}$. The increase of $\delta^{*}$ with $U$ follows the trend found for the location of the Sordi transition [86].

At finite temperature in the presence of interactions, Fig. 1 also shows dashed lines in the $T-\delta$ plane that indicate the 

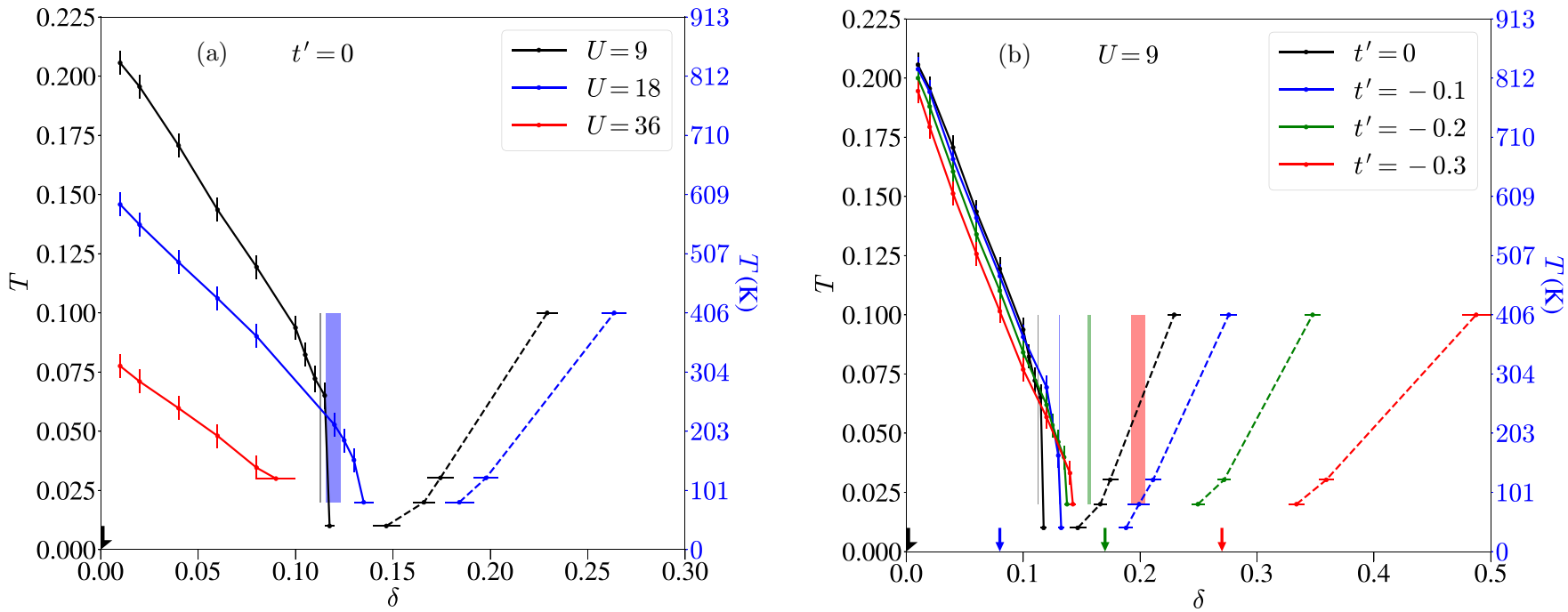

FIG. 1. For a given value of $U$ at $t^{\prime}=0$ (left) or finite $t^{\prime}$ at $U=9$ (right), the pseudogap $T^{*}$ line is represented by a solid line, the van Hove line by a dashed line, and the zone of maximum entropy by a solid rectangle. The arrows indicate the positions of the van Hove singularities in the noninteracting case for different $t^{\prime}$. Severe sign problems sometimes occurred at low temperature or large doping, especially for $U=36$. The blue temperature axis was obtained from $t \simeq 350 \mathrm{meV}$.

maximum of the single-particle local density of states at the Fermi level. This is not the usual noninteracting van Hove singularity, but it is adiabatically connected to it. We see on the figure that the dashed lines [the van Hove lines $T_{\mathrm{VH}}(\delta)$ ] are pushed to higher doping compared to the location of the van Hove singularity in the noninteracting case (arrows). Consequently, the experimental conjecture Eq. (1) for $\delta_{\text {phase }}^{*}$ seems, in our calculations, to extend to $\delta^{*}$ since $\delta^{*} \leqslant \delta_{\mathrm{VH}}$.

The location of the zone of maximum of entropy in the $T-\delta$ plane, represented by a colored rectangle, is also pushed by interactions to higher doping. The displacement of the zone of maximum entropy towards higher doping $\delta$ with increasing $U$ would be consistent with its coincidence with the end of the coexistence region of the Sordi transition [85], whose $\delta$ also increases with $U$. This coincidence has, however, been proven only for $U=6.2$.

It seems paradoxical that with increasing $U$ the $T^{*}$ line extends to higher doping but appears at lower temperatures. This comes from the antagonistic effects of $U$ on singlet formation. On the one hand, local magnetic moments increase with $U$, leading to better-defined singlets at low temperature. On the other hand, the decrease of $J$ with increasing $U$ makes these singlets less resilient to thermal fluctuations.

Figure 1 (right) shows the normal-state phase diagram at $U=9$ for $t^{\prime}=0,-0.1,-0.2$, and -0.3 . The dependence on $t^{\prime}$ of $\delta^{*}$ follows the trend found for the location of the Sordi transition (see Supplemental Material of Ref. [86]). Remarkably, two main experimental observations regarding $\delta_{\text {phase }}^{*}$ and $T_{\text {phase }}^{*}(\delta)$ are also found for $\delta^{*}$ and $T^{*}(\delta)$ in this figure. Indeed, one experiment [25] finds that changing the value of $t^{\prime}$ through chemical pressure does not affect the initial slope of the $T_{\text {phase }}^{*}$ line at low doping but $\delta_{\text {phase }}^{*}$ monotonically moves towards higher doping with increasing values of $\left|t^{\prime}\right|$. Moreover, another experiment [26] shows that applied pressure pushes $\delta_{\text {phase }}^{*}$ to lower doping while calculations in the same paper [26] find a concomitant decrease of $\left|t^{\prime}\right|$.
Interactions push the van Hove line $T_{\mathrm{VH}}(\delta)$ to higher doping than the noninteracting van Hove singularity, as in the $t^{\prime}=0$ case. The effect of $t^{\prime}$ at fixed $U$ agrees with Ref. [83]: The larger $\left|t^{\prime}\right|$, the less $U$ pushes the van Hove line away from the noninteracting case (colored arrows in Fig. 1). This is consistent with the decreasing influence of interactions at large doping. Besides, the experimental conjecture Eq. (1) for $\delta_{\text {phase }}^{*}$ extends once again to $\delta^{*}$ as $\delta^{*} \leqslant \delta_{\mathrm{VH}}$. Finally, the zone of maximum entropy also moves towards higher doping with increasing $\left|t^{\prime}\right|$. More importantly, it is farther and farther away from both the Widom and the van Hove lines, as suggested in Ref. [96].

\section{B. Critical scaling of the specific heat}

Let us finally discuss critical scaling of the specific heat. We computed the total energy [86] and fitted it with $E_{\text {tot }}=$ $a+b T^{2} \ln T+c T^{2}$. The specific heat is the derivative of the fitted total energy with respect to temperature, yielding $C / T=2 b \ln T+(b+2 c)$, presented on a semilog plot in Fig. 2 for $U=9, t^{\prime}=0$ (left) and for $U=9, t^{\prime}=-0.3$ (right). Since the goodness of the energy fit holds over a wide range of dopings, as shown in the Supplemental Material [93], so does the critical scaling of $C / T$. A typical cuprate hopping amplitude of $t \simeq 350 \mathrm{meV}$ converts $C / T$ to a value that is of the same order as that found experimentally [24]. However, our lowest temperature $T=0.01 t$ is about $40 \mathrm{~K}$. This minimal temperature is much higher than the experimental range $0.5-10 \mathrm{~K}$ in Ref. [24] where a critical fan with a sharp peak in the doping dependence of $C / T$ at fixed $T$ appears. Instead, we find that at higher temperature the value of $C / T$ is essentially the same for a wide range of parameters around the critical point $\delta^{*} \sim 0.12$ for $t^{\prime}=0$ and $\delta^{*} \sim 0.15$ for $t^{\prime}=-0.3$ that we determined from $T^{*}$.

This observation can be understood by taking experimental studies in heavy fermions $[97,98]$ as typical examples of what is expected for the quantum critical behavior of $C / T$. Heavy 

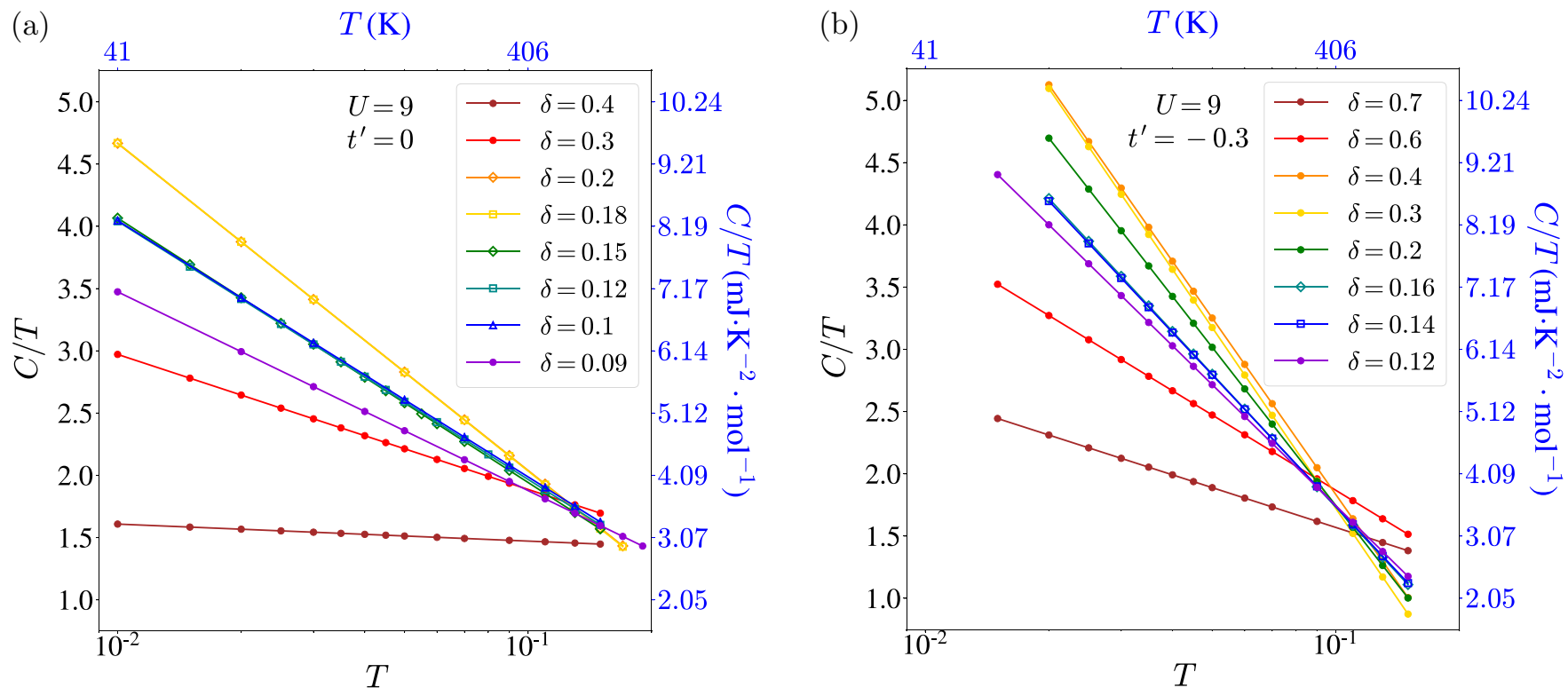

FIG. 2. Specific heat divided by temperature as a function of temperature for different dopings at $U=9, t^{\prime}=0$ (left) and $U=9, t^{\prime}=-0.3$ (right). After extracting the total energy of the system and fitting it as $E_{\mathrm{tot}}=a+b T^{2} \ln T+c T^{2}$, the specific heat is extracted as its derivative with respect to temperature, yielding $C / T=2 b \ln T+(b+2 c)$. The solid lines are guides to the eye. The temperature in degrees Kelvin and specific heat axes (in blue) were generated using $t \simeq 350 \mathrm{meV}$.

fermions exhibit an extremely low effective Fermi energy so that the high-temperature limit of the electronic specific heat is accessible without phonon contamination. It is found that at high temperature $C / T$ is indeed logarithmic and very weakly dependent on the parameters. The equivalence established in Ref. [26] between applying pressure in experiments and decreasing $\left|t^{\prime}\right|$ in calculations enables us to establish another analogy with Refs. [97,98]: At a given doping, the slope of $C / T$ as a function of temperature on the semilog plot becomes flatter with decreasing $\left|t^{\prime}\right|$, as we find by comparing the two plots in Fig. 2 [99]. When doping increases beyond the critical doping, $C / T$ again becomes insensitive to doping for a small doping range, reflecting the presence of the van Hove line $T_{\mathrm{VH}}(\delta)$. Upon increasing doping sufficiently, $C / T$ gradually becomes temperature independent, as expected in a Fermi liquid.

\section{CONCLUSION}

In summary, consistent with most earlier theoretical studies, we find that the NMR pseudogap $T^{*}$ line [100] shares many features of the experimental $T_{\text {phase }}^{*}$ line [21,24-26,3134]: (a) $T^{*}$ drops precipitously to zero at a doping $\delta^{*}$. (b) $T^{*}$ near half filling scales as superexchange $J=4 t^{2} / U$ at large $U$. (c) Changes in the band structure modify primarily the value of $\delta^{*}$ and it is possible to see this effect experimentally by applying pressure. (d) The doping where the van Hove line extrapolates at low temperature, $\delta_{\mathrm{VH}}(T \rightarrow 0)$, seems to satisfy the inequality (1) in the $T=0$ limit. (e) The high-temperature specific heat is consistent with expectations for high-temperature quantum critical behavior. (f) The doping where entropy is maximum is almost independent of $T$.

Our contribution for theory is that the quantum critical behavior of the electronic specific heat is connected to doped
Mott-insulating behavior. Indeed, the extrapolated position of the quantum critical point seems to have the same dependence on $t^{\prime}$ as the Sordi transition, which is continuously connected to the Mott transition at half filling. The criticalpoint temperature for the Sordi transition is known to decrease rapidly with increasing $U$ and should be investigated further.

Additional experiments on the NMR $T^{*}$ are called for to verify our observation that the $T^{*}$ line is a necessary condition for the appearance of the experimental $T_{\text {phase }}^{*}$ line. That $T_{\text {phase }}^{*}$ line occurs at lower temperature and is thus more sensitive to details of the Hamiltonian. Another prediction for experiment is that the doping where entropy is maximum depends strongly on the band parameters, an effect that could be measured with combined specific-heat and pressure experiments.

\section{ACKNOWLEDGMENTS}

We are grateful to S. Verret and M.-H. Julien for useful discussions, to O. Simard for help, and to Louis Taillefer for comments on the manuscript. The work of P.S. was supported by the U.S. Department of Energy, Office of Science, Basic Energy Sciences as a part of the Computational Materials Science Program. This research was undertaken thanks in part to funding from the Canada First Research Excellence Fund. This work has been supported by the Natural Sciences and Engineering Research Council of Canada (NSERC) under Grant No. RGPIN-2014-04584, and by the Research Chair in the Theory of Quantum Materials. Simulations were performed on computers provided by the Canadian Foundation for Innovation, the Ministère de l'Éducation des Loisirs et du Sport (Québec), Calcul Québec, and Compute Canada. 
[1] H. Alloul, T. Ohno, and P. Mendels, Phys. Rev. Lett. 63, 1700 (1989).

[2] C. Berthier, M. H. Julien, M. Horvatić, and Y. Berthier, J. Phys. I France 6, 2205 (1996).

[3] N. J. Curro, T. Imai, C. P. Slichter, and B. Dabrowski, Phys. Rev. B 56, 877 (1997).

[4] T. Timusk and B. Statt, Rep. Prog. Phys. 62, 61 (1999).

[5] S. Kawasaki, C. Lin, P. L. Kuhns, A. P. Reyes, and G.-q. Zheng, Phys. Rev. Lett. 105, 137002 (2010).

[6] B. Fauqué, Y. Sidis, V. Hinkov, S. Pailhès, C. T. Lin, X. Chaud, and P. Bourges, Phys. Rev. Lett. 96, 197001 (2006).

[7] H. A. Mook, Y. Sidis, B. Fauque, V. Baledent, and P. Bourges, Phys. Rev. B 78, 020506(R) (2008).

[8] L. Mangin-Thro, Y. Sidis, A. Wildes, and P. Bourges, Nat. Commun. 6, 7705 (2015).

[9] R. Daou, J. Chang, D. LeBoeuf, O. Cyr-Choiniere, F. Laliberte, N. Doiron-Leyraud, B. J. Ramshaw, R. Liang, D. A. Bonn, W. N. Hardy, and L. Taillefer, Nature (London) 463, 519 (2010).

[10] A. Shekhter, B. J. Ramshaw, R. Liang, W. N. Hardy, D. A. Bonn, F. F. Balakirev, R. D. McDonald, J. B. Betts, S. C. Riggs, and A. Migliori, Nature (London) 498, 75 (2013).

[11] Y. Lubashevsky, L. D. Pan, T. Kirzhner, G. Koren, and N. P. Armitage, Phys. Rev. Lett. 112, 147001 (2014).

[12] L. Zhao, C. A. Belvin, R. Liang, D. A. Bonn, W. N. Hardy, N. P. Armitage, and D. Hsieh, Nat. Phys. 13, 250 (2017).

[13] C. V. Parker, P. Aynajian, E. H. da Silva Neto, A. Pushp, S. Ono, J. Wen, Z. Xu, G. Gu, and A. Yazdani, Nature (London) 468, 677 (2010).

[14] G. Sordi, P. Sémon, K. Haule, and A.-M. S. Tremblay, Sci. Rep. 2, 547 (2012).

[15] An eight-site DCA calculation of the Knight shift [74,83] for a smaller value of $U$ than that considered here nevertheless gives a temperature dependence of the $T^{*}$ line quite comparable to ours.

[16] G. Sordi, K. Haule, and A.-M. S. Tremblay, Phys. Rev. Lett. 104, 226402 (2010).

[17] T. D. Stanescu and P. Phillips, Phys. Rev. Lett. 91, 017002 (2003).

[18] S. Badoux, W. Tabis, F. Laliberté, G. Grissonnanche, B. Vignolle, D. Vignolles, J. Béard, D. A. Bonn, W. N. Hardy, R. Liang, N. Doiron-Leyraud, L. Taillefer, and C. Proust, Nature (London) 531, 210 (2016).

[19] F. Laliberté, W. Tabis, S. Badoux, B. Vignolle, D. Destraz, N. Momono, T. Kurosawa, K. Yamada, H. Takagi, N. DoironLeyraud, C. Proust, and L. Taillefer, arXiv:1606.04491.

[20] C. Collignon, S. Badoux, S. A. A. Afshar, B. Michon, F. Laliberté, O. Cyr-Choinière, J.-S. Zhou, S. Licciardello, S. Wiedmann, N. Doiron-Leyraud, and L. Taillefer, Phys. Rev. B 95, 224517 (2017).

[21] B. Loret, S. Sakai, S. Benhabib, Y. Gallais, M. Cazayous, M. A. Méasson, R. D. Zhong, J. Schneeloch, G. D. Gu, A. Forget, D. Colson, I. Paul, M. Civelli, and A. Sacuto, Phys. Rev. B 96, 094525 (2017).

[22] Y. Ando, Y. Kurita, S. Komiya, S. Ono, and K. Segawa, Phys. Rev. Lett. 92, 197001 (2004).

[23] A. P. Mackenzie, S. R. Julian, D. C. Sinclair, and C. T. Lin, Phys. Rev. B 53, 5848 (1996).

[24] B. Michon, C. Girod, S. Badoux, J. Kacmarcík, Q. Ma, M. Dragomir, H. A. Dabkowska, B. D. Gaulin, J.-S. Zhou,
S. Pyon, T. Takayama, H. Takagi, S. Verret, N. DoironLeyraud, C. Marcenat, L. Taillefer, and T. Klein, Nature (London) 567, 218 (2019).

[25] O. Cyr-Choinière, R. Daou, F. Laliberté, C. Collignon, S. Badoux, D. LeBoeuf, J. Chang, B. J. Ramshaw, D. A. Bonn, W. N. Hardy, R. Liang, J.-Q. Yan, J.-G. Cheng, J.-S. Zhou, J. B. Goodenough, S. Pyon, T. Takayama, H. Takagi, N. Doiron-Leyraud, and L. Taillefer, Phys. Rev. B 97, 064502 (2018).

[26] N. Doiron-Leyraud, O. Cyr-Choinière, S. Badoux, A. Ataei, C. Collignon, A. Gourgout, S. Dufour-Beauséjour, F. F. Tafti, F. Laliberté, M.-E. Boulanger, M. Matusiak, D. Graf, M. Kim, J.-S. Zhou, N. Momono, T. Kurosawa, H. Takagi, and L. Taillefer, Nat. Commun. 8, 2044 (2017).

[27] T. Kondo, T. Takeuchi, T. Yokoya, S. Tsuda, S. Shin, and U. Mizutani, J. Electron Spectrosc. Relat. Phenom. 137, 663 (2004).

[28] J. Chang, M. Shi, S. Pailhés, M. Månsson, T. Claesson, O. Tjernberg, A. Bendounan, Y. Sassa, L. Patthey, N. Momono, M. Oda, M. Ido, S. Guerrero, C. Mudry, and J. Mesot, Phys. Rev. B 78, 205103 (2008).

[29] T. Yoshida, M. Hashimoto, S. Ideta, A. Fujimori, K. Tanaka, N. Mannella, Z. Hussain, Z.-X. Shen, M. Kubota, K. Ono, S. Komiya, Y. Ando, H. Eisaki, and S. Uchida, Phys. Rev. Lett. 103, 037004 (2009).

[30] C. E. Matt, C. G. Fatuzzo, Y. Sassa, M. Månsson, S. Fatale, V. Bitetta, X. Shi, S. Pailhès, M. H. Berntsen, T. Kurosawa, M. Oda, N. Momono, O. J. Lipscombe, S. M. Hayden, J.-Q. Yan, J.-S. Zhou, J. B. Goodenough, S. Pyon, T. Takayama, H. Takagi, L. Patthey, A. Bendounan, E. Razzoli, M. Shi, N. C. Plumb, M. Radovic, M. Grioni, J. Mesot, O. Tjernberg, and J. Chang, Phys. Rev. B 92, 134524 (2015).

[31] J. Tallon and J. Loram, Physica C: Superconductivity 349, 53 (2001).

[32] J. Tallon, T. Benseman, G. Williams, and J. Loram, Physica C: Superconductivity 415, 9 (2004).

[33] J. G. Storey, J. L. Tallon, G. V. M. Williams, and J. W. Loram, Phys. Rev. B 76, 060502(R) (2007).

[34] J. L. Tallon (unpublished).

[35] S. Chakraborty, D. Galanakis, and P. Phillips, Phys. Rev. B 82, 214503 (2010).

[36] A. Garg, B. S. Shastry, K. B. Dave, and P. Phillips, New J. Phys. 13, 083032 (2011)

[37] G. Grissonnanche, O. Cyr-Choinière, F. Laliberté, S. René de Cotret, A. Juneau-Fecteau, S. Dufour-Beauséjour, M.-È. Delage, D. LeBoeuf, J. Chang, B. J. Ramshaw, D. A. Bonn, W. N. Hardy, R. Liang, S. Adachi, N. E. Hussey, B. Vignolle, C. Proust, M. Sutherland, S. Krämer, J.-H. Park, D. Graf, N. Doiron-Leyraud, and L. Taillefer, Nat. Commun. 5, 3280 (2014).

[38] G. Sordi, P. Sémon, K. Haule, and A.-M. S. Tremblay, Phys. Rev. Lett. 108, 216401 (2012).

[39] K.-S. Chen, Z. Y. Meng, S.-X. Yang, T. Pruschke, J. Moreno, and M. Jarrell, Phys. Rev. B 88, 245110 (2013).

[40] E. Gull, O. Parcollet, and A. J. Millis, Phys. Rev. Lett. 110, 216405 (2013).

[41] T. Nakano, M. Oda, C. Manabe, N. Momono, Y. Miura, and M. Ido, Phys. Rev. B 49, 16000 (1994).

[42] M. C. Gutzwiller, Phys. Rev. Lett. 10, 159 (1963).

[43] J. Hubbard, Proc. R. Soc. London, Ser. A 276, 238 (1963). 
[44] J. Kanamori, Prog. Theor. Phys. 30, 275 (1963).

[45] T. Maier, M. Jarrell, T. Pruschke, and M. H. Hettler, Rev. Mod. Phys. 77, 1027 (2005).

[46] G. Kotliar, S. Y. Savrasov, K. Haule, V. S. Oudovenko, O. Parcollet, and C. A. Marianetti, Rev. Mod. Phys. 78, 865 (2006).

[47] A.-M. S. Tremblay, B. Kyung, and D. Sénéchal, Low Temp. Phys. 32, 424 (2006).

[48] J. E. Hirsch and R. M. Fye, Phys. Rev. Lett. 56, 2521 (1986).

[49] A. N. Rubtsov, V. V. Savkin, and A. I. Lichtenstein, Phys. Rev. B 72, 035122 (2005).

[50] E. Gull, A. J. Millis, A. I. Lichtenstein, A. N. Rubtsov, M. Troyer, and P. Werner, Rev. Mod. Phys. 83, 349 (2011).

[51] P. Werner and A. J. Millis, Phys. Rev. B 74, 155107 (2006).

[52] P. Werner, A. Comanac, L. de Medici, M. Troyer, and A. J. Millis, Phys. Rev. Lett. 97, 076405 (2006).

[53] K. Haule, Phys. Rev. B 75, 155113 (2007).

[54] E. Gull, P. Werner, A. Millis, and M. Troyer, Phys. Rev. B 76, 235123 (2007)

[55] E. Gull, P. Werner, O. Parcollet, and M. Troyer, Europhys. Lett. 82, 57003 (2008).

[56] A. I. Lichtenstein and M. I. Katsnelson, Phys. Rev. B 62, R9283 (2000).

[57] M. Jarrell, T. Maier, M. H. Hettler, and A. N. Tahvildarzadeh, Europhys. Lett. 56, 563 (2001).

[58] M. Civelli, M. Capone, S. S. Kancharla, O. Parcollet, and G. Kotliar, Phys. Rev. Lett. 95, 106402 (2005).

[59] A. Macridin, M. Jarrell, T. Maier, P. R. C. Kent, and E. D’Azevedo, Phys. Rev. Lett. 97, 036401 (2006).

[60] B. Kyung, S. S. Kancharla, D. Sénéchal, A.-M. S. Tremblay, M. Civelli, and G. Kotliar, Phys. Rev. B 73, 165114 (2006).

[61] K. Haule and G. Kotliar, Phys. Rev. B 76, 104509 (2007).

[62] M. Ferrero, P. S. Cornaglia, L. De Leo, O. Parcollet, G. Kotliar, and A. Georges, Phys. Rev. B 80, 064501 (2009).

[63] E. Gull, O. Parcollet, P. Werner, and A. J. Millis, Phys. Rev. B 80, 245102 (2009).

[64] S. Sakai, Y. Motome, and M. Imada, Phys. Rev. Lett. 102, 056404 (2009).

[65] P. Werner, E. Gull, O. Parcollet, and A. J. Millis, Phys. Rev. B 80, 045120 (2009).

[66] E. Gull, M. Ferrero, O. Parcollet, A. Georges, and A. J. Millis, Phys. Rev. B 82, 155101 (2010).

[67] O. Gunnarsson, T. Schäfer, J. P. F. LeBlanc, E. Gull, J. Merino, G. Sangiovanni, G. Rohringer, and A. Toschi, Phys. Rev. Lett. 114, 236402 (2015).

[68] K. Haule, A. Rosch, J. Kroha, and P. Wölfle, Phys. Rev. Lett. 89, 236402 (2002).

[69] K. Haule, A. Rosch, J. Kroha, and P. Wölfle, Phys. Rev. B 68, 155119 (2003).

[70] N. Bittner, D. Golež, H. U. R. Strand, M. Eckstein, and P. Werner, Phys. Rev. B 97, 235125 (2018).

[71] K. Mikelsons, E. Khatami, D. Galanakis, A. Macridin, J. Moreno, and M. Jarrell, Phys. Rev. B 80, 140505(R) (2009).

[72] N. S. Vidhyadhiraja, A. Macridin, C. Şen, M. Jarrell, and M. Ma, Phys. Rev. Lett. 102, 206407 (2009).

[73] T. A. Maier, P. Staar, V. Mishra, U. Chatterjee, J. C. Campuzano, and D. J. Scalapino, Nat. Commun. 7, 11875 (2016).

[74] X. Chen, J. P. F. LeBlanc, and E. Gull, Nat. Commun. 8, 14986 (2017).
[75] D. J. Scalapino, E. Loh, and J. E. Hirsch, Phys. Rev. B 34, 8190 (1986)

[76] T. A. Maier, D. Poilblanc, and D. J. Scalapino, Phys. Rev. Lett. 100, 237001 (2008).

[77] T. Dahm, V. Hinkov, S. V. Borisenko, A. A. Kordyuk, V. B. Zabolotnyy, J. Fink, B. Büchner, D. J. Scalapino, W. Hanke, and B. Keimer, Nat. Phys. 5, 217 (2009).

[78] B. Kyung, D. Sénéchal, and A.-M. S. Tremblay, Phys. Rev. B 80, 205109 (2009)

[79] D. J. Scalapino, Rev. Mod. Phys. 84, 1383 (2012).

[80] S. Nishiyama, K. Miyake, and C. M. Varma, Phys. Rev. B 88, 014510 (2013).

[81] D. Sénéchal, A. G. R. Day, V. Bouliane, and A.-M. S. Tremblay, Phys. Rev. B 87, 075123 (2013).

[82] A. Reymbaut, M. Charlebois, M. F. Asiani, L. Fratino, P. Sémon, G. Sordi, and A.-M. S. Tremblay, Phys. Rev. B 94, 155146 (2016).

[83] W. Wu, M. S. Scheurer, S. Chatterjee, S. Sachdev, A. Georges, and M. Ferrero, Phys. Rev. X 8, 021048 (2018).

[84] H. Bragança, S. Sakai, M. C. O. Aguiar, and M. Civelli, Phys. Rev. Lett. 120, 067002 (2018).

[85] G. Sordi, K. Haule, and A.-M. S. Tremblay, Phys. Rev. B 84, 075161 (2011).

[86] L. Fratino, P. Sémon, G. Sordi, and A.-M. S. Tremblay, Sci. Rep. 6, 22715 (2016).

[87] L. Xu, P. Kumar, S. V. Buldyrev, S.-H. Chen, P. H. Poole, F. Sciortino, and H. E. Stanley, Proc. Natl. Acad. Sci. USA 102, 16558 (2005)

[88] H. Oike, K. Miyagawa, H. Taniguchi, and K. Kanoda, Phys. Rev. Lett. 114, 067002 (2015).

[89] G. Sordi, P. Sémon, K. Haule, and A.-M. S. Tremblay, Phys. Rev. B 87, 041101(R) (2013).

[90] L. Fratino, P. Sémon, G. Sordi, and A.-M. S. Tremblay, Phys. Rev. B 93, 245147 (2016).

[91] G. Kotliar, S. Y. Savrasov, G. Pálsson, and G. Biroli, Phys. Rev. Lett. 87, 186401 (2001).

[92] S. Sakai, G. Sangiovanni, M. Civelli, Y. Motome, K. Held, and M. Imada, Phys. Rev. B 85, 035102 (2012).

[93] See Supplemental Material at http://link.aps.org/supplemental/ 10.1103/PhysRevResearch.1.023015 for an explanation of how we find $T^{*}(\delta)$ and $\delta^{*}$ from the magnetic susceptibility, how we do analytic continuation to find the van Hove line $T_{\mathrm{VH}}(\delta)$, how we use a Maxwell relation to find the location of the maximum of entropy, and how we find the specific heat. We also explain how error bars are estimated.

[94] T. A. Maier and D. J. Scalapino, arXiv:1810.10043.

[95] D. C. Johnston, Phys. Rev. Lett. 62, 957 (1989).

[96] G. Sordi (unpublished).

[97] H. von Löhneysen, J. Phys.: Condens. Matter 8, 9689 (1996).

[98] H. v. Löhneysen, A. Rosch, M. Vojta, and P. Wölfle, Rev. Mod. Phys. 79, 1015 (2007).

[99] The logarithmic temperature dependence of $C / T$ is also found in the two-impurity Kondo problem [101] where longwavelength spin fluctuations are unimportant. The singlets that are found in the pseudogap phase of plaquette CDMFT calculations $[14,61]$ may lead to similar behavior.

[100] In Bi2201, the NMR $T^{*}$ [5] seems to coincide with $T_{\text {phase }}^{*}$ as determined from ARPES and resistivity.

[101] I. Affleck and A. W. W. Ludwig, Phys. Rev. Lett. 68, 1046 (1992). 